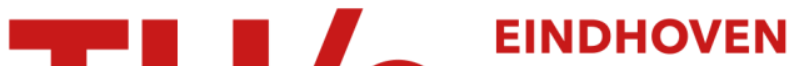

\section{Understanding activity scheduling and rescheduling behaviour : theory and numerical illustration}

\section{Citation for published version (APA):}

Joh, C. H., Arentze, T. A., \& Timmermans, H. J. P. (2001). Understanding activity scheduling and rescheduling behaviour : theory and numerical illustration. GeoJournal, 53(4), 359-371.

https://doi.org/10.1023/A:1020145214526

DOI:

10.1023/A:1020145214526

Document status and date:

Published: 01/01/2001

\section{Document Version:}

Publisher's PDF, also known as Version of Record (includes final page, issue and volume numbers)

\section{Please check the document version of this publication:}

- A submitted manuscript is the version of the article upon submission and before peer-review. There can be important differences between the submitted version and the official published version of record. People interested in the research are advised to contact the author for the final version of the publication, or visit the $\mathrm{DOI}$ to the publisher's website.

- The final author version and the galley proof are versions of the publication after peer review.

- The final published version features the final layout of the paper including the volume, issue and page numbers.

Link to publication

\section{General rights}

Copyright and moral rights for the publications made accessible in the public portal are retained by the authors and/or other copyright owners and it is a condition of accessing publications that users recognise and abide by the legal requirements associated with these rights.

- Users may download and print one copy of any publication from the public portal for the purpose of private study or research.

- You may not further distribute the material or use it for any profit-making activity or commercial gain

- You may freely distribute the URL identifying the publication in the public portal.

If the publication is distributed under the terms of Article 25fa of the Dutch Copyright Act, indicated by the "Taverne" license above, please follow below link for the End User Agreement:

www.tue.nl/taverne

Take down policy

If you believe that this document breaches copyright please contact us at:

openaccess@tue.nl

providing details and we will investigate your claim. 


\title{
Understanding activity scheduling and rescheduling behaviour: Theory and numerical illustration
}

\author{
Chang-Hyeon Joh, Theo A. Arentze \& Harry J.P. Timmermans \\ Urban Planning Group, Eindhoven University of Technology, P.O. Box 513, 5600 MB Eindhoven, The Netherlands
}

Key words: travel demand modelling, activity-based approach, (re)scheduling, utility function, decision heuristics

\section{Introduction}

Inspired by Hagerstrand's time geography, the activity-based modelling approach has become an active area of research since the mid 90s in transportation research (Ettema and Timmermans, 1997). As a consequence, it has regained interest in geography as well. The central concept underlying the activity-based approach is that travel is derived from the participation in activities instead of being pursued for its own sake, and therefore, the understanding, analysis and forecasting of travel behaviour should be based on the understanding of activities (Burnett and Hanson, 1982). Individuals try to meet their personal and family needs by participating in activities in everyday life, subject to a set of constraints. In the process of organising activities in time and space, travel is derived as a by-product to overcome the distance between activity locations. Any direct causation of socio-demographic characteristics and the physical environment to travel behaviour without explicitly considering the choice of activity participation may, therefore, be incorrect or at least theoretically inappropriate.

The last two decades have witnessed an enormous amount of literature that illustrates a variety of research directions in this paradigm. Two distinctive approaches should be mentioned here. First, the utility maximisation approach assumes that individuals choose the best solution through the search of all possible alternatives. Key studies in this direction include Bowman and Ben-Akiva (2000), Fujii et al. (1998), McNally (1997), Recker et al. (1985) and Recker (1995). As pointed out in Gärling et al. (1994), however, while the utility maximisation principle might explain which factors affect the final choice, it does not account for the process of making decisions that also impact on outcomes. If the goal is to forecast travel demand, this may not be an urgent research question. It is however important for a better understanding of travel behaviour.

Secondly, to overcome this limitation, the computational process approach, which assumes that individuals pursue satisfactory, possibly sub-optimal, solutions through the search of a subset of the universe of alternatives, has been introduced. This approach focuses on the decision making process and offers more insight into how people process information and arrive at the observed activity-travel pattern. Because of its focus on the decision process, this approach potentially allows one to better evaluate the implications of various transportation policy measures (e.g., Bhat and Koppelman, 2000). Examples include Arentze et al. (2000), Ettema et al. (1993) and Gärling et al. (1998). Among these, Arentze et al. (2000) presented the latest and most comprehensive operational model to date, named Albatross, a rule based system, which derives choice heuristics from diary data and predicts activity-travel patterns.

Yet, research to date has paid little attention to the decision making process underlying activity schedule changes under time pressure and unexpected events. Everyday life is full of uncertainty, and frequently, individuals are forced to reconsider previously scheduled activities. In fact, scheduling and rescheduling are a never-ending process over the entire lifetime of individuals and are complementary to each other. Scheduling is an incremental process that gradually increases the level of detail of the sequence of activities as activities are executed in a given time horizon. Doherty and Miller (2000) found in a week-long survey of activity scheduling in Canada that $60 \%$ of modifications and $41 \%$ of cancellations of activities were made during the execution of the schedule.

Although Gärling et al. (1999) have undertaken pioneering work on this topic and provided a principle of schedule change where people delete or postpone particular activities under time pressure, they only considered some selected aspects of rescheduling behaviour. The authors, therefore developed a more comprehensive model of scheduling and rescheduling behaviour. It includes the theoretical foundation of a short-term dynamics model of activity-travel behaviour (Timmermans et al., 2001), the elaborated utility functions (Joh et al., 2001), and the complementary model component representing decision heuristics (Joh et al., 2002). As a continuation of this modelling work, the current research aims to examine whether the model exhibits the envisioned activity behaviour as a function of some important model parameters. To this end, the current paper simulates initial scheduling and time pressure-induced rescheduling behaviour as conducted by different hypothetical individuals characterised by different parameters. 


\section{The model}

Our theory of individuals' scheduling and rescheduling behaviour involves the following conceptual considerations. First, individuals execute activities to meet a variety of needs. Fulfilling activities returns satisfaction or utility as a reward for meeting the needs. The list of activities to conduct is determined by an individual's personal desires, responsibility for family and work contract (Damm and Lerman, 1981; Bhat and Koppelman, 1993).

Secondly, a set of circumstantial conditions limits the extent to which individuals can increase the utility. These conditions include individuals' physical condition, their role in society and the physical environment surrounding them. These conditions encourage individuals and society to develop particular ways or means of fulfilling activities. Activities of particular purposes are then organised in space and time (Pred, 1981; Thrift, 1983).

Thirdly, uncertainty should also be taken into account. Given the needs and conditions, individuals identify and evaluate activities in terms of their anticipated utilities for possible implementation. Uncertainty, however, affects the evaluation due to the fact that the activities in the later positions of the planned schedule may involve a larger amount of uncertainty. The evaluation results differ between individuals faced with uncertainty, dependent on their personalities or decision styles in dealing with uncertainty, which in turn affect the schedule.

Fourthly, individuals are assumed to use heuristics in looking for alternatives instead of becoming involved in an exhaustive search, due to the fact that their rationality is bounded. Individuals usually have numerous alternative ways of planning a schedule given a time horizon, each of which may result in a different level of utility. Cognitive constraints however prevent individuals from identifying and evaluating every single one of a universe of alternatives. Individuals therefore use a set of heuristics to reduce the burden of search and to pursue cost effectiveness. A typical example is habitual behaviour (Gärling, 1998) that does not concern any other alternatives than a routinised alternative, which is far from optimising behaviour but is frequently the case. Heuristic behaviour may result in sub-optimal, satisfying choices.

Finally, an activity schedule is tentative and may be changed at any time. Every moment in time, there may be the need for changing the schedule of remaining not-yetcompleted activities. An individual may be forced to change the schedule due to time pressure or may actively decide to change to improve the existing schedule. Any (sub-optimal) decision is enforced until a further need to reschedule the activities arises.

Based on the above discussion, we formulate a conceptual framework of individuals' scheduling and rescheduling behaviour as illustrated in Figure 1. Initially, a tentative schedule is given. The set of activities included in the current schedule is a subset of the activity program. The individual evaluates the utility of activities for possible implementation as well as non-implementation. When an individual with a

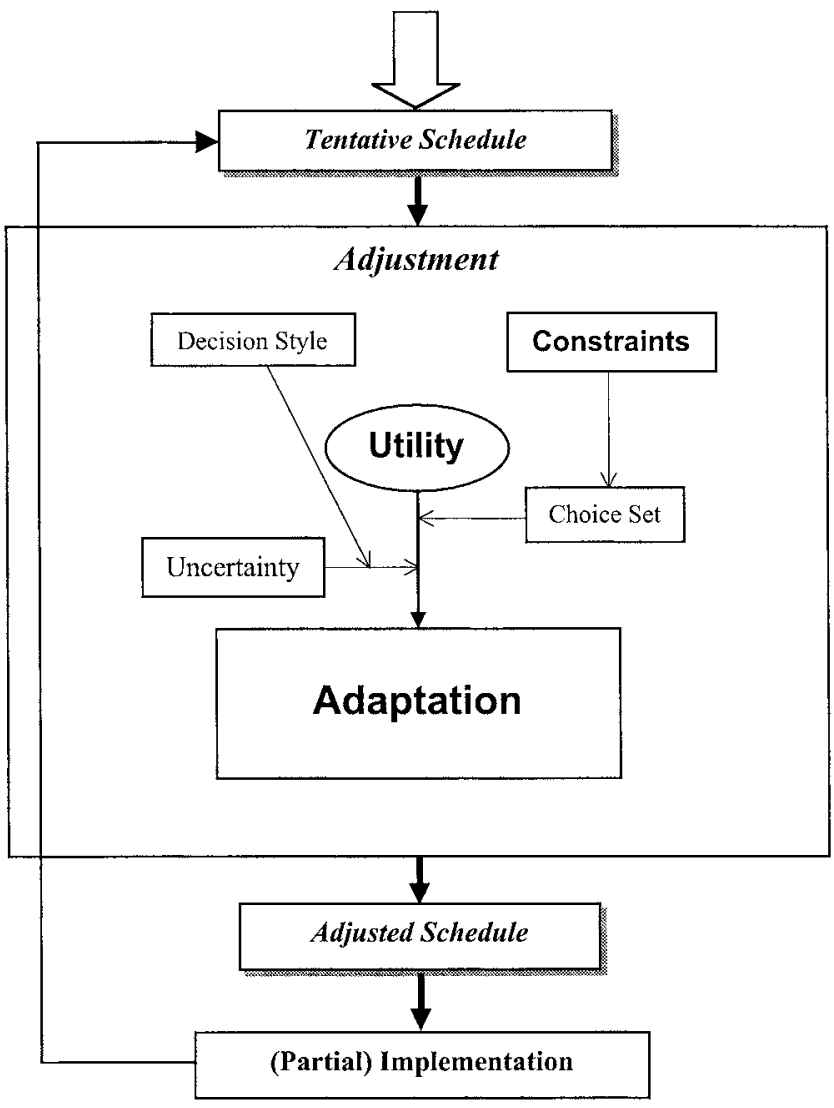

Figure 1. Coneptual framework.

certain decision style evaluates the utility of alternative activities under a set of constraints, he/she examines whether some change of the schedule is necessary. More specifically, the individual examines whether there is any time pressure or any improvement of utility level is possible by changing the existing schedule. Because it is impossible to identify and evaluate all possible alternatives due to cognitive constraints, individuals adopt certain heuristic strategies to effectively and efficiently reduce the search space to reach reasonably good solutions in real time. The adjusted activity schedule then is implemented. The utility of the remaining schedule is again subject to unexpected events causing increased or reduced time pressure. Therefore, the schedule will often be only partially implemented, and the adjusted schedule remains tentative.

In the following sub-sections we provide a formal description of two major components of the suggested model: the utility functions and the heuristic solution methods.

\section{The utility function}

Individuals derive a certain level of utility directly or indirectly from participating in activities. The utility function associates some selected characteristics of activities with particular numeric values that are assumed to correspond to particular utility levels. The utility also varies with characteristics of individuals and households. We assume that the utility of an activity is a function of the amount of time spent on the activity, where longer duration provides a higher level of utility. 
Microeconomic theory assumes an increasing utility function with a diminishing marginal utility over the entire range of input variable values. The utility functions of activities used in activity-based approaches adopting timeuse microeconomic theory (Becker, 1965) almost invariably rely on the same assumption of an ever-diminishing marginal utility over the entire range of activity duration (Kitamura et al., 1996), although many other functional forms are conceivable, as argued by Supernak (1988). We introduced such an alternative utility function (Timmermans et al., 2001), paying particular attention to the possibility of increasing marginal utility due to a negative saturation effect in the beginning phase of the implementation of certain activities. Many leisure activities and some initial information-acquisition activities such as shopping activity possibly display this property.

Here, we reintroduce the proposed functional form of the utility function and provide a detailed formal description of its properties. Although utility is primarily a function of the time spent on the concerned activity, many other choice facets such as location, transport mode, etc. also affect the utility that individuals derive from becoming involved in an activity. Furthermore, as mentioned earlier, uncertainty impacts utility, dependent on decision styles and the process of schedule adjustment. In this subsection, however, we do not concern ourselves with this variety of choice facets and uncertainty-related behaviour, but focus only on the assumed utility function under certainty. Readers interested in other choice facets and uncertainty are referred to Joh et al., (2001).

Let $A P$ refer to the given activity program, $A P=$ $\{1, \ldots, A\}$. The proposed utility function can be written as:

$$
U_{a}=U_{a}^{\min }+\frac{U_{a}^{\max }-U_{a}^{\min }}{\left(1+\exp \left[-\beta_{a}\left(v_{a}-\alpha_{a}\right)\right]\right)^{\gamma_{a}}},
$$

where:

$a \quad$ is an index of activities $(a \in A P)$;

$v \quad$ is the duration $(v \geq 0)$;

$U^{\max } \quad$ is the upper asymptote of the curve $\left(U^{\max }>0\right)$;

$U^{\text {min }} \quad$ is the lower asymptote of the curve $\left(U^{\min } \leq 0\right)$;

$\alpha \quad$ is parameter of the location of the curve $(\alpha>0)$;

$\beta \quad$ is parameter of the slope of the curve $(\beta>0)$;

$\gamma \quad$ is parameter of the inflection point of the curve $(\gamma>0)$.

Equation (1) dictates that the utility of an activity is a function of duration with a set of activity-specific parameters. In particular, the utility function in Equation (1) has the following properties. First, it is a monotonically increasing function of duration since:

$\frac{d U}{d v}=\frac{\gamma \beta\left(U^{\max }-U^{\min }\right)}{\exp [\beta(v-\alpha)](1+\exp [-\beta(\nu-\alpha)])^{\gamma+1}}>0$.

This implies that the utility of an activity is primarily determined by the amount of duration spent on the activity and that a longer duration gives a higher utility.

Secondly, utility is however not infinitely increasing but converges to a maximum level as:

$$
\lim _{\nu \rightarrow \infty} U=U^{\max }
$$

The maximum level of utility differs between activities.

Thirdly, the minimum utility or zero-duration utility is given by:

$$
U_{\nu=0}=U^{\min }+\frac{U^{\max }-U^{\min }}{(1+\exp [\alpha \beta])^{\gamma}}
$$

The size of $U_{\nu=0}$ may differ between activities. A zero or approximately zero value is considered the normal case. However, for some activities the value may be negative. For example, not conducting a leisure activity may produce fatigue or other intrinsic negative effects that reduces total utility for the schedule as a whole. A large negative utility would facilitate the inclusion of the concerned activity. On the other hand, a positive value would not be consistent with the theory and, therefore, would avoid imposing this constraint on the values the parameters can take.

Fourthly, the utility function is asymmetrically S-shaped, and the marginal utility increases up to a certain level of utility (inflection point) and decreases afterwards, i.e.:

$$
\begin{aligned}
\frac{d^{2} U}{d \nu^{2}}= & \frac{\gamma \beta^{2}\left(U^{\mathrm{max}}-U^{\min }\right)(1+\exp [-\beta(\nu-\alpha)])^{r}(\gamma-\exp [\beta(\nu-\alpha)])}{\exp [2 \beta(\nu-\alpha)](1+\exp [-\beta(\nu-\alpha)])^{2 \gamma+2}} \\
& \geqq 0
\end{aligned}
$$

and the inflection point is given as $U\left(v^{*}\right)$ where $v^{*}=$ $\frac{\alpha \beta+\ln \gamma}{\beta}$. The utility function has a concave part as well as a convex part. A larger $\gamma$ causes a lower utility at the inflection point, $U\left(v^{*}\right)=U^{\min }+\frac{U^{\max }-U^{\min }}{\left(1+\gamma^{-1}\right)^{\gamma}}$, as $\frac{\partial U\left(v^{*}\right)}{\partial \gamma}<0$, and a smaller proportion of increasing marginal utility. The particular values of $U\left(v^{*}\right)$ may vary across activities and individuals, and can be captured by estimating the $\gamma$ value.

Finally, the activation and the speed of utility change vary with parameters $\alpha$ and $\beta$, respectively. As implied by Equation (1), $\alpha$ and $\beta$ determine the location of the utility function along the duration axis and the slope of the curve, respectively. The change in utility takes place after a certain amount of time, and the amount that activates the change varies across activities. An increasing $\alpha$ shifts the curve to the right, meaning that more time is needed to reach a particular utility level. The speed of utility change over time also varies across activities. The curve is steeper with a larger $\beta$, implying that the change in utility is more sensitive to the change in duration, keeping everything else constant.

Given the utility function for individual activities, the total function for an entire schedule should aggregate the utilities across activities. The aggregation equation can be manifold, and some multiplicative form may be desirable to reflect the mutual relationships between activities. Obviously, certain activities have complementary or substitution relationships. A business meeting, for example, has a complementary relationship with a follow-up social event. Inhome and out-of-home leisure activities are likely to have a substitution relationship because of the fixed amount of time in the evening after work. To cope with these activityspecific relationships, the constituent activities should be grouped in a multiplicative term of an additive aggregate function. In the current study however we simplify the problem and assume a simple additive aggregate utility function: 


$$
U=\sum_{a=1}^{A} U_{a}
$$

with

$$
\sum_{a=1}^{A} v_{a}=B
$$

where $B$ is the total duration ( $B>0$ ), representing the budget constraint that limits the total amount of time that can be assigned to activities at the moment of scheduling.

The additive form with the time budget constraint implies that activities have a general relationship with all others regarding their duration in the schedule. Increasing the duration of an activity means without exception that the duration of other activities is decreased, depending on their utility function. To increase total utility, therefore, the increase of utility for the extended activitie(s) must exceed the utility decrease of the reduced activitie(s). Othewise, no change is induced. Secondly, however, an activity does not have any activity-specific relationship with other activities.

\section{Decision heuristics}

Models predicting the choice of a complete activity schedule (Jones et al., 1983; Recker et al., 1985) typically generate all feasible schedules and select the best one. In contrast, models of incremental activity scheduling behaviour (Gärling et al., 1998; Ettema et al., 1993) employ a set of operators and heuristic rules that control the application of those operators and induce incremental adjustments of the schedule. Incremental adjustment implies that the schedule is changed in a stepwise manner and the adjustment of the current step is made on and hence affected by the current state of the schedule.

We adopt the latter modelling approach. That is, we employ a set of operators including duration adjustment and insertion, deletion and substitution operators to implement incremental adjustments of the schedule. (We should mention again that other operators that might be related to choice facets such as location and transport-mode are not considered here.) The duration operator adjusts the duration of the scheduled activities such that the marginal utility becomes the same across the activities included in the schedule, and the system reaches an equilibrium state. Insertion, deletion and substitution operators change the list of scheduled activities such that unscheduled activities are added to the schedule, and scheduled activities are removed if this increases the total utility.
Among these operators, only the duration operator involves a fine-tuning of duration and realises the equalisation of the marginal utilities across scheduled activities. All three other operators involve discontinuous adjustments in which sudden larger changes in duration takes place, and the resulting schedule may well be out of equilibrium. Note that the operators are considered only if the adjustments meet the schedule constraints. The envisioned scheduling and rescheduling behaviour is therefore highly discontinuous due to constraints that limit feasible adjustments.

We assume the following decision heuristics. First, at each time of incremental adjustment, an individual mentally simulates the schedule change implied by each operator, and chooses the best operator that offers the highest increase in total utility. Secondly, the mental simulation of an operator requires an effort and discounts utility, the amount of which is constant and specific to each operator. This effort can be interpreted as operator-specific resistance to change. Likewise, any actual implementation of schedule change by the chosen operator counts as effort and reduces the total utility, the amount of which is an increasing function of the number of adjustment steps up to the current adjustment. This effort can be regarded as cumulative mental fatigue. Finally, these adjustment processes continue until no improvement of the schedule is possible. In other words, if the highest increase of total utility is negative, the system stops the adjustment process.

The evaluation is initiated at the beginning of a day for an initial schedule and resumes at any time of necessity including the completion of an activity and the occurrence of an unexpected event. Let $S$ and $R$ be the set of activities that are currently scheduled and not scheduled yet, respectively, implying $S \cup R=A P$ and $S \cap R=\phi$. Let $O$ be a subset of operators, $O=\left\{O_{A}, O_{I}, O_{D}, O_{S}\right\}$, where $O_{A}$ denotes duration adjustment, $O_{I}$ insertion, $O_{D}$ deletion and $O_{S}$ substitution. Given the suggested utility function and heuristics, scheduling and rescheduling behaviour is represented as an algorithm below.

Thus, the system first calculates for each operator $o$ the amount of improvement $\left(\Delta U_{t-1}^{o}\right)$, computed as the incremental total utility $\left(\tilde{U}_{t-1}^{o}-U_{t-1}\right)$, discounted by resistance to change $\left(\theta_{o}\right)$ and mental fatigue cumulated up to the current adjustment step $(h(t))$. Next, the system selects the operator $\left(o_{t}\right)$ that will return the highest utility increase at the current adjustment step. If the total utility after adjustment is higher than the existing schedule's utility, the system implements the adjustment using the chosen operator $\left(S_{t} \leftarrow A P_{t-1}^{o}\right)$. Note that operators are applied not only on $S_{t-1}$ but also on $R_{t-1}$ because of insertion of activities into $S$ from $R$ and deletion of activities from $S$ into $R$. The member activities of $S$ and $R$ may differ between $A P_{t-1}$ and $A P_{t}$. Finally, the termination condition is expressed as the non-improvement of the existing schedule above the fatigue and resistance threshold. 


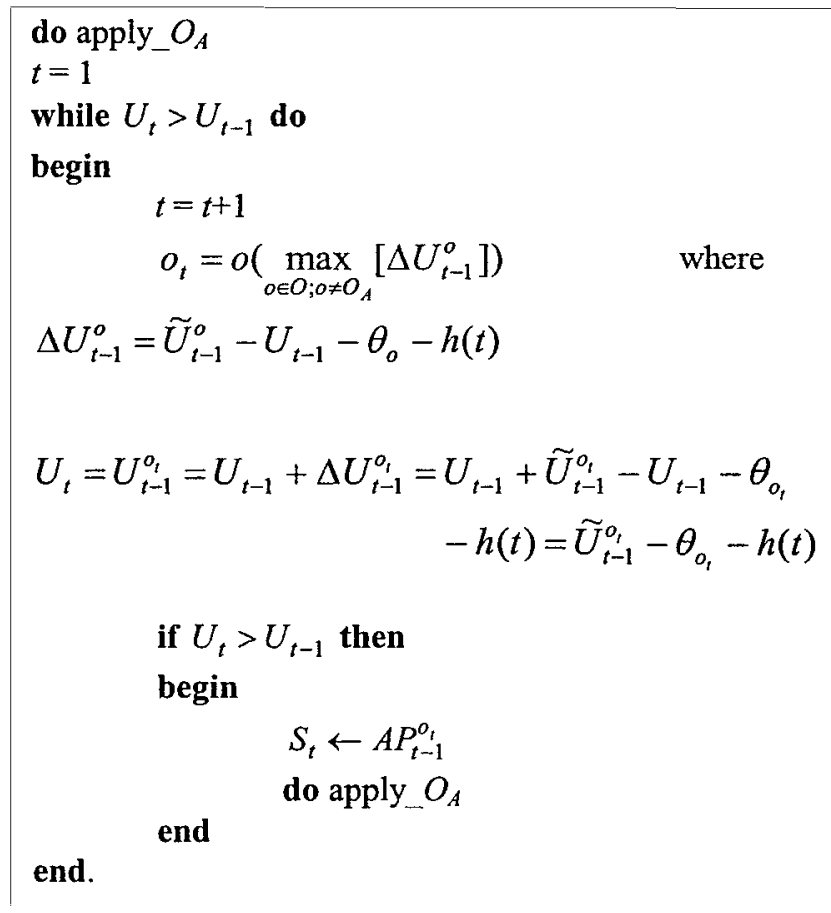

where:

$t \quad$ is an index of adjustment steps $(t>0)$;

$o \quad$ is an index of operators;

$S_{t} \quad$ is $S$ at adjustment step $t$, where $S_{0}$ implies the initial schedule;

$U_{t-1} \quad$ is total utility of $A P_{t-1}$;

$\tilde{U}_{t-1}^{o} \quad$ is non-discounted total utility when applying operator $o$ on $A P_{t-1}$.

$\theta_{o} \quad$ is the level of resistance to change by operator $o\left(\theta_{o} \geq 0\right)$;

$h(t) \quad$ is the cumulative mental fatigue at adjustment step $t$;

$\Delta U_{t-1}^{o} \quad$ is the increment of $U_{t-1}$ discounted by $\theta_{o}$ and $h(t)$ when applying operator $o$ on $A P_{t-1}$.

Throughout the adjustment processes, the duration adjustment operator plays an important role that supports other operators. The activities of the initial schedule are not likely to have an equal marginal utility, so that a fine-tuning of duration between activities to increase total utility is needed. Schedule adjustment by other operators thereafter likely disturbs this duration equilibrium. The duration operator is then again activated to get the schedule back into the equilibrium state.

\section{An examination of scheduling and rescheduling behaviour}

We examine scheduling and rescheduling behaviour by varying some selected utility parameters representing individuals' characteristics. In particular, we simulate two different values for respectively $U^{\max }, \beta$ and $\gamma$ of certain activities and investigate the impact of the different parameter values on scheduling and rescheduling behaviour. Note that the focus of the current paper is to study the impact of utility parameters given the decision heuristics. No variation of heuristics parameters is introduced. The system keeps all resistance to change and mental fatigue parameters constant to zero $\left(\theta_{o}=0 \forall o\right.$, and $\left.h(t)=0 \forall t\right)$ throughout the simulations. In the following subsections, we first describe the different parameter settings used in the simulations, then discuss expected results, and finally, describe the results of the simulation.

\section{Simulation settings}

The simulation consists of two parts: initial scheduling and rescheduling induced by increased time pressure. Hypothetical full-time workers who are characterised by particular values of utility parameters are given an initial schedule that consists only of skeleton activities including sleep, work and in-home leisure. In the first part of the simulation, each individual gradually optimises the schedule. In the second part, a time pressure situation is imposed on the individuals that forces them to change their previously optimised schedules. The simulated time pressure situation is the consequence of congestion on the road to the first out-of-home activity in the morning. The duration of travel is increased by half an hour, and individuals clear the time pressure and further adjust the schedule.

Table 1 provides the list of activities of the activity program and the utility parameter values. A total of ten activities is included in the activity program. Out-of-home leisure, daily shopping and dinner activities respectively have two alternative values of $U^{\max }, \beta$ and $\gamma$. Each of the eight combinations of parameter values $(2 \times 2 \times 2)$ characterises an individual. Table 2 shows the initial schedule where only the sequence and duration of the skeleton activities are specified. The current simulation does not consider the location attribute. Therefore, the classification of in-home and outof-home activities follows an arbitrary definition. Table 3 represents the assumed time constraints in terms of facility opening hours and work contract hours.

\section{Individual characteristics and simulation focus}

Table 1 identifies the following eight distinctive combinations of individual characteristics related to out-of-home leisure, daily shopping and dinner activities.

Hypothetical person 1: High Leis_O $U^{\max }$, large Dshop $\beta$, and large Dinner $\gamma$.

Hypothetical person 2: High Leis_O $U^{\max }$, large Dshop $\beta$, and small Dinner $\gamma$.

Hypothetical person 3: High Leis_O $U^{\max }$, small Dshop $\beta$, and large Dinner $\gamma$.

Hypothetical person 4: High Leis_O $U^{\max }$, small Dshop $\beta$, and small Dinner $\gamma$.

Hypothetical person 5: Low Leis_O $U^{\max }$, large Dshop $\beta$, and large Dinner $\gamma$.

Hypothetical person 6: Low Leis_O $U^{\max }$, large Dshop $\beta$, 
Table 1 Hypothetical activity programme and parameters.

\begin{tabular}{lrrrrll}
\hline $\begin{array}{l}\text { Activity } \\
\text { in AP }\end{array}$ & $E D$ & $U^{\min }$ & $U^{\max }$ & \multicolumn{1}{c}{$\alpha$} & $\beta$ & $\gamma$ \\
\hline Sleep & 360 & -5 & 200 & 400 & 0.16 & 2.5 \\
Pcare & 10 & 0 & 130 & 55 & 0.5 & 5 \\
Work1 & 180 & -5 & 250 & 150 & 0.15 & 5 \\
Lunch & 30 & 0 & 135 & 35 & 0.15 & 5 \\
Work2 & 300 & -5 & 250 & 250 & 0.15 & 1 \\
Dinner & 30 & 0 & 160 & 55 & 0.1 & 5 \\
Leis_I & 360 & 0 & 60 & 100 & 0.1 & 1 \\
Leis_O & 60 & 0 & 210 & 100 & 0.1 & 1 \\
Mcare & 30 & 0 & 100 & 65 & 0.425 & 1 \\
Dshop & 10 & 0 & 125 & 35 & 0.5 & 5 \\
& & & & & 0.01 & 5 \\
\hline
\end{tabular}

Pcare, Work1, Work2, Leis_I, Leis_O, Mcare and Dshop respectively denote personal care, work in the morning, work in the afternoon, in-home leisure, out-of-home leisure, medical care and daily shopping. $E D$ represents some expected duration of an activity that is assumed as given. The cells in grey denote the parameter variation in the current simulation.

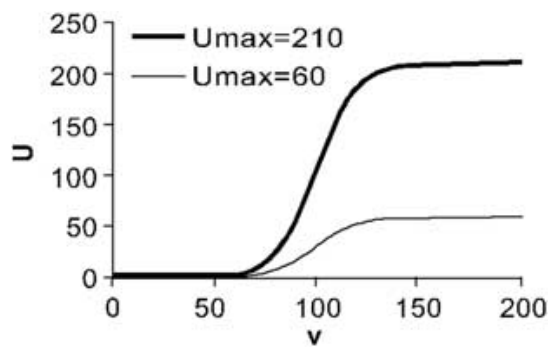

Figure 2. Utility of Leis_O activity varying with $U^{\max }$

and small Dinner $\gamma$.

Hypothetical person 7: Low Leis_O $U^{\max }$, small Dshop $\beta$, and large Dinner $\gamma$.

Hypothetical person 8: Low Leis_O $U^{\max }$, small Dshop $\beta$, and small Dinner $\gamma$.

The identification of eight hypothetical persons with different characteristics implies that the system will optimise eight times the same initial schedule and will also implement eight times a congestion-induced rescheduling situation on the previously optimised schedules. Note that the initial schedule optimisation will be conducted on the same initial schedule across simulations of different parameter settings,

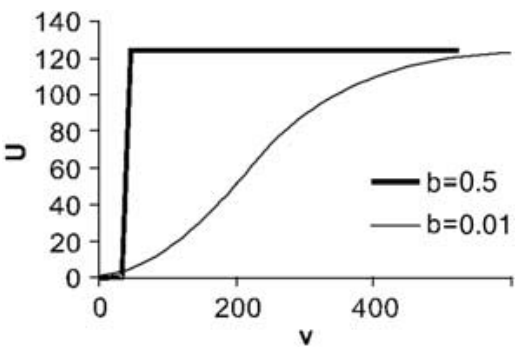

Figure 3. Utility of Dshop activity varying with $\beta$.

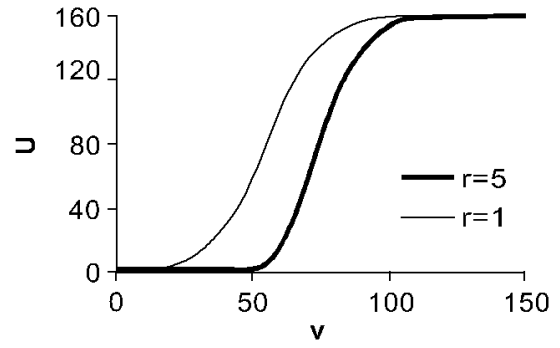

Figure 4. Utility of Dinner activity varying with $\gamma$.

while the rescheduling simulations will be conducted on the schedules that likely differ between parameter settings. In the following, we discuss the expected results of scheduling and rescheduling behaviour, dependent on these parameter settings.

Given the utility parameter settings provided in Table 1, we expect the following impacts. First, a high $U^{\text {max }}$ of Leis_O implies that one has a higher utility when conducting leisure outside home (see Figure 2). This may prompt the person to spend more time on leisure activities outside home. Secondly, a large $\beta$ of Dshop as reflected in Figure 3 implies that one reaches the maximum utility very quickly after some amount of time spent for daily shopping. Because in case of a large $\beta$ only a small range of duration shows meaningful changes of utility, the duration of daily shopping is probably also limited across schedules. In case of a small $\beta$, on the other hand, the utility function is very flat and shows smaller utility differences between durations, which probably induces a wide variation of duration across schedules. Furthermore, the curve with a small $\beta$ provides a lower level of utility for most of the duration range. Finally, Figures 4 and 5 show that a large $\gamma$ provides a much lower level of utility for the same duration, a very steep slope, while most part of utility function is increasing with diminishing marginality. This means that the relevant activity requires a longer duration to reach the near maximum utility. Once that level is reached, an individual is reluctant to reduce the duration of this activity because of the steeper slope and the larger loss of marginal utility.

Based on these expected impacts of each of the concerned parameter values, we postulate the following scheduling and rescheduling behaviour for each individual. Person 1 will spend more time on outside leisure activities and dinner and less time on daily shopping, while Person 2 would spend more time on outside leisure activities and less time on daily shopping and dinner. Person 3 is expected to spend more time on outside leisure activities and dinner, while the amount of time for daily shopping of flat-slope utility function would depend on the amount of time spent for other activities. Likewise, Person 4's amount of time spent on daily shopping will also depend on the amount of time for other activities, while spending more time on outside leisure activities and less time on dinner. Person 5 and 6 are all expected to spend less time on outside leisure activities and daily shopping, while more time on dinner for Person 5 and less time on dinner for Person 6. Person 7 may spend less time on leisure activities and more time on dinner, 
Table 2 Initial schedule.

\begin{tabular}{|c|c|c|c|}
\hline Activity & Duration & Begin time & End time \\
\hline Sleep & 480 & $0: 00$ & $8: 00$ \\
\hline * & - & - & - \\
\hline Workl & 180 & 900 & 12.00 \\
\hline$*$ & - & - & - \\
\hline Work2 & 300 & 13.00 & 18.00 \\
\hline * & - & - & - \\
\hline Leis_I & 300 & $19: 00$ & $24: 00$ \\
\hline
\end{tabular}

\begin{tabular}{|c|c|c|c|}
\hline Activity & Duration & Begin time & End time \\
\hline Pcare & 0 & - & - \\
\hline Dinner & 0 & - & - \\
\hline Lunch & G & 3 & $=$ \\
\hline Lers 0 & 0 & 4 & He \\
\hline Meare & 0 & ? & - \\
\hline Dshop & 0 & 4 & 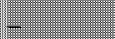 \\
\hline
\end{tabular}

In the activity column, "*' denotes that activity is not defined for the time slot yet. Activities having zero duration are those activities not included in the initial schedule. Activities in grey are assumed out-of-home, while other activities in-home.

Table 3 Institutional constraints (opening hours and work contract).

\begin{tabular}{lllll}
\hline Activity & $\begin{array}{l}\text { Earliest possible } \\
\text { begin time }\end{array}$ & $\begin{array}{l}\text { Latest possible } \\
\text { begin time }\end{array}$ & $\begin{array}{l}\text { Earliest possible } \\
\text { end time }\end{array}$ & $\begin{array}{l}\text { Latest possible } \\
\text { end time }\end{array}$ \\
\hline Work1 & - & $09: 30$ & $11: 30$ & $12: 30$ \\
Work2 & $12: 30$ & $13: 30$ & $17: 30$ & - \\
Mcare & $10: 00$ & - & - & $18: 00$ \\
Dshop & $08: 00$ & - & - & $20: 00$ \\
\hline
\end{tabular}

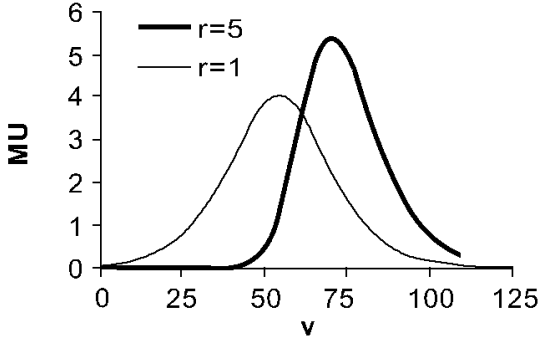

Figure 5. Marginal utility of Dinner activity.

while Person 8 less time on dinner. The amount of time for daily shopping will be dependent upon the amount of time for other activities in case of Person 7 and 8.

\section{Results}

Here, the simulated schedule adjustments will be compared for utility parameter settings with regard to the following aspects of activity-travel behaviour.

- The number of activities in the final schedule compared to the initial schedule

- The number of adjustment steps

- The list of operations used for the adjustments

- Whether or not the activities varying the parameters $U^{\max }, \beta$ and $\gamma$ are included in the final schedule

- The change in the duration distribution across activities before and after the adjustments

- The ratio of the duration of out-of-home to in-home activities in the final schedule

- The total utility of the final schedule

Initial optimisation of the schedule. Overall, the application of operators (the sequence, number and list of operators) was almost the same across settings, and most adjustment processes involved duration and insertion operators. This is because the initial optimisation of the schedule with a large amount of open time slots naturally includes many insertions of activities with a certain duration and duration adjustment after each insertion. However, much diversity existed in the initial optimisation of the schedule, depending on the scheduling parameters of the activities (see Appendix I).

First, $U^{\text {max }}$ of Leis_O had the following impacts. (1) Leis_O with a higher $U^{\max }$ was always included in the schedule across $\beta$ s and $\gamma \mathrm{s}$, while a lower $U^{\max }$ failed to include Leis_O in most cases. Because Leis_O's duration for reaching $U^{\text {max }}$ was relatively long, the inclusion of this outof-home activity reduced the duration of other out-of-home activities. More specifically, when Leis_O was included, the duration of work was reduced. (2) Closely related to this result, a higher $U^{\max }$ induced a bigger ratio of the duration of out-of-home activities to the duration of in-home activities. This is because a lower $U^{\max }$ of outside leisure activities are in most cases substituted with inside leisure activities and dinner. (3) More importantly, the difference in utility is already very large between Leis_Os with higher and lower $U^{\max }$ as seen in Figure 2. As a result, a higher $U^{\max }$ resulted in a much higher level of total utility.

Secondly, the $\beta$ parameter for the daily shopping activities had the following impacts. (1) Daily shopping activities with a larger $\beta$ tend to have a fixed amount of time. This is because Dshop with a larger $\beta$ shows a sharp increase of utility in the range of 30 to 50 duration units and, if included, should have a duration near 50 and should not be affected too much by other schedule circumstances (see Figure 3 ). Daily shopping activities with a smaller $\beta$, on the other hand, show a slow increase of utility over the range up to 600 and are more strongly affected by the state of other activities in the schedule, resulting in the deletion of daily shopping activities in several cases. (2) A daily shopping activity with a large $\beta$ was included in all final schedules across the settings. This can be explained by the fact that a relatively short duration adds a reasonable amount of utility to the total utility 
without requiring other activities to be reduced much. (3) A larger $\beta$ for daily shopping activity clearly induces a higher level of total utility. If $\beta$ is large, the utility approximates its maximum; a smaller $\beta$ provides a utility much lower than the maximum. Even if the duration is longer with for example Person 3 (53 units) than Person 1 (48 units), this episode is not long enough to approximate the maximum utility at all, as seen in Figure 3.

Finally, $\gamma$ for dinner activity provided the following impacts. (1) Dinner was included in all final schedules across settings due to a high maximum utility with moderate duration. (2) The unitary value of the $\gamma$ parameter $(=1)$ provides a typical symmetric $S$-shaped utility function. Amplifying $\gamma$ has the effect of pushing the inflection point down, while keeping the duration with the maximum utility unchanged. As shown in Figure 4, the utility curve then has a smaller portion of increasing marginal utility and a larger portion of diminishing marginal utility from the viewpoint of utility axis (y-axis). Consequently, dinner activity with a large value of $\gamma(>1)$ comes closer to the conventional utility curve of diminishing marginal utility. Figure 5 shows that dinner activity with a unitary value of $\gamma$ has a symmetric change of marginal utility with duration, while dinner activity with a larger $\gamma$ has an asymmetric change that has a rapid increase up to the inflection point and a slow decrease afterwards. (3) A larger $\gamma$ with everything else equal lowers the level of utility over the entire range of duration up to the point of maximum utility (see Figure 4). In other words, dinner activity with a larger $\gamma$ requires a longer duration than dinner activity with a smaller $\gamma$ to achieve the same level of utility. Consequently, a larger $\gamma$ induced a longer duration of dinner activity, while providing a lower level of total utility. This is because a shorter duration of dinner activity with a smaller $\gamma$ is already greater in utility than a longer duration of dinner activity with a larger $\gamma$. Furthermore, a shorter duration of dinner activity with a smaller $\gamma$ enables other activities to have longer duration, which in turn increases total utility.

Rescheduling after congestion. Overall, rescheduling involved a much smaller number of adjustments, compared to the initial scheduling, because the given schedules were previously optimised, and the adjustment was conducted mainly around the activity directly related to the time pressure scenario. Nevertheless, rescheduling behaviour clearly illustrated the amendments of previous scheduling decisions and involved subsequent deletion and insertion operations. Furthermore, besides the envisioned impacts of the simulated parameter values, the given initial states of the previously optimised schedules that differ between parameter settings provide a significant difference in rescheduling behaviour (see Appendix II).

The time pressure scenario is superimposed on a schedule that is in an (optimised) equilibrium state. It assumes that congestion, or any external force, will effectively reduce the optimised duration of a particular activity. If this reduction significantly disrupts the equilibrium state, the system will engage in a rescheduling procedure. In the current simula- tion, the time pressure scenario imposed congestion on the way to work in the morning, which caused 30 units longer travel time than usual.

The primary determinant of whether the imposed time pressure is significant enough to move the system in a rescheduling mode was the state of the activity that was directly related to the time pressure rather than the different settings of scheduling parameter values. This does however not mean that the scheduling parameters representing personal characteristics of individuals are not important to the rescheduling of activities. The result of rescheduling (list of activities, activity sequence and duration) was very much dependent on the schedule that was given by the initial optimisation. Therefore, while the rescheduling process itself was not very different between settings, the final schedules were quite different.

Person 2 had the initial optimised schedule where daily shopping activity with a larger $\beta$ was conducted on the way to work after sleep activity was conducted at home. Their rescheduling resulted in a significant increase of the duration of daily shopping activity, and consequently, a big increase in total utility. The explanation for this result is that the system first removed daily shopping activity of a too short duration on the way to work and inserted it again with a longer duration to another position after the second work activity. As a consequence of this increase in the duration of daily shopping activity, the near zero utility level of this activity increased to a near maximum utility level, which resulted in the increased total utility. Likewise, when the first work episode was scheduled after in-home activities in the morning and its reduced duration was much smaller than 180 units, rescheduling involved a substantial increase in utility by increasing the duration of the first work activity, while changing the duration of other activities. Persons 4 and 8 exhibit this rescheduling pattern. This was, however, not the case when the reduced duration of the work activity was close to 180 units as in the cases of Persons 1, 3, 5, 6 and 7 (Figure 6).

It follows from the above discussion that rescheduling choices strongly depended on the initial state of the optimised schedule. The impacts of different parameter settings can be summarised as follows. First, $U^{\max }$ had the following impact. (1) The increase in $U^{\max }$ enhances the possibility of the occurrence and long duration of the corresponding activity in the final schedule. (2) In case of an out-of-home activity, an increase in $U^{\max }$ increases the possibility of a higher ratio of out-of-home activity to in-home activity duration. (3) An increase in $U^{\max }$ generally results in a higher total utility value.

Secondly, $\beta$ had the following impact. (1) A larger $\beta$ for daily shopping activity results in the duration that is often the same across simulations, while a smaller $\beta$ likely induces diversity in the duration. This outcome arises because most utility changes occur between the minimum utility and maximum utility duration, and this effective range is very small for an activity with a larger $\beta$. This, therefore, explains an almost fixed amount of duration for an activity with a larger $\beta$. (2) An activity with a larger $\beta$ had a much higher utility 


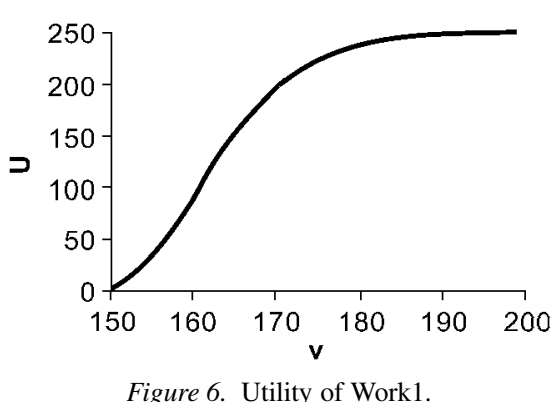

level than an activity with a smaller $\beta$ over a wide range of duration, and the final schedule including an activity with a larger $\beta$, in general provides a higher level of total utility, keeping everything else equal. (3) If the duration of an activity implied by the maximum utility is short compared to other activities, the size of $\beta$ does not show a clear impact on the ratio of the out-of-home duration to the in-home duration in the final schedule.

Finally, $\gamma$ showed the following impacts. (1) Because, with everything else equal, an activity with a larger $\gamma$ faces an earlier and sharper decrease of utility when the duration is reduced from that of the maximum utility, an activity with a larger $\gamma$ tends to have a longer duration to avoid this effect. (2) The final schedule including an activity with a smaller $\gamma$ likely to have a higher level of total utility because a smaller $\gamma$ has a higher level of utility than a bigger $\gamma$ over the entire range of duration. This result in turn implies that, with everything else equal, an activity with a smaller $\gamma$ requires a shorter duration to achieve the same level of utility, which increases the possibility of longer duration of other activities in the schedule. (3) Interestingly, the difference in the ratio of the out-of-home duration between larger and smaller $\gamma \mathrm{s}$ of an activity shows an interrelationship with the size of the $U^{\max }$ parameter. When $U^{\max }$ is large, outside leisure activities are included across the final schedules. A larger $\gamma$ of dinner activity that results in a longer duration of the activity then implies that the schedule is not able to further include inside leisure activities. A smaller $\gamma$ that results in a shorter duration of dinner activity on the other hand induces the inclusion of inside leisure activities in the schedule. Leisure activities have bigger units of duration than dinner activity, and consequently, a larger $\gamma$ results in a bigger ratio of out-of-home duration compared to a larger $\gamma$, given a larger $U^{\max }$. When $U^{\max }$ is small, outside leisure activities are mostly excluded, and dinner and inside leisure activities constitute the schedule after work. Given this situation, a larger $\gamma$ of dinner activity that results in a longer duration of the activity directly induces a smaller ratio of out-of-home duration.

\section{Conclusion}

This article has described a model of activity scheduling and rescheduling behaviour under time pressure and examined the behaviour of the model in terms of a set of selected parameters. The model consists of two main components: a utility function and a set of decision heuristics. The assumed utility function of activity duration is S-shaped and able to represent a variety of changes in marginal utility over time. Decision heuristics involve a set of operators including duration adjustment, insertion, deletion and substitution. The heuristics mimic an individual's (re)scheduling behaviour that is sometimes continuous (duration operator) but most of the time highly discontinuous due to the non-incremental adjustments of insertion, deletion and substitution operators and the constraints that introduces discontinuous choice sets to the model.

The simulation consisted of two subsequent parts, i.e. optimisation of an initial schedule and rescheduling under time pressure. Particular values of selected parameters were varied to study their behaviour. As theorised, we found that activities with higher maximum utilities and higher inflection points are more likely to provide a higher level of total utility in the final schedule. Secondly, with everything else equal, an activity's utility function with a steeper slope results in almost the same duration across simulation settings while that with a flatter slope may result in various lengths of duration depending on other activities' duration. Consequently, an activity with a flatter slope is more likely to have a lower level of total utility in the final schedule when the resulting duration of that activity is long. Finally, the imposed time pressure situation shows that, besides the impact of the parameter settings, the state of the previously optimised initial schedule is of crucial importance to the overall process of rescheduling. If the currently given optimised schedule was much in conflict with the imposed situation of time pressure, the hypothetical individuals are forced to conduct a substantial adjustment to the schedule. Overall then, it has been shown that different parameter settings characterising different individuals resulted in distinguishable, envisioned, state dependent activity scheduling and rescheduling behaviour. This implies that once the necessary parameters are obtained via some estimation procedure, the suggested model of short-term dynamics of scheduling and rescheduling behaviour can be applied to the understanding, analysis and forecasting of the activity-travel behaviour in the real world.

\section{Acknowledgements}

The research, reported in this paper, is conducted as part of the Amadeus research programme sponsored by the Dutch Research Foundation (NWO). The details of the project are reported in Arentze et al. (2001).

\section{References}

Arentze T.A., Hofman F., Van Mourik H. and Timmermans H.J.P. 2000: Albatross: A multi-agent rule-based model of activity pattern decisions. Transportation Research Record, 1706: 136-144.

Arentze T., Dijst M., Dugundji E., Joh C.H., Kapoen L., Krijgsman S., Maat K., Timmermans H. and Veldhuisen J. 2001: The Amadeus program: Scope and conceptual development. Paper presented at the 9th World Conference on Transportation Research, Seoul, July.

Bhat C.R. and Koppelman F.S. 1993: A conceptual framework of individual activity program generation. Transportation Research A, 27: 433-446. 
Bhat C.R. and Koppelman F.S. 2000: Activity-based travel demand analysis: History, results and future directions. Paper presented at the 79th Annual Meeting of the Transportation Research Board, Washington DC, January.

Bowman J.L. and Ben-Akiva M.E. 2000: Activity-based disaggregate travel demand model system with activity schedules. Transportation Research A, 35: 1-28.

Burnett P. and Hanson S. 1982: The analysis of travel as an example of complex human behaviour in spatially-constrained situations: Definition and measurement issues. Transportation Research A, 16: 87-102.

Damm D. and Lerman S.R. 1981: A theory of activity scheduling behaviour Environment and Planning A, 13: 703-718.

Doherty S.T. and Miller E.J. 2000: A computerised household activity scheduling behaviour. Transportation, 27: 75-97.

Ettema D., Borgers A. and Timmermans H. 1993: A simulation model of activity scheduling behaviour. Transportation Research Record, 1413: $1-11$.

Ettema D. and Timmermans H. 1997: Theories and models of activity-travel patterns. In: Ettema D. and Timmermans H.J.P. (eds) Activity-based Approaches to Travel Analyses. Pergamon, Oxford, pp. 1-36.

Fujii S., Kitamura R. and Monma T. 1998: A utility-based micro-simulation model system of individuals' activity-travel patterns. Paper presented at the 77th Annual Meeting of the Transportation Research Board, Washington D.C., January.

Gärling T., Kwan M.P. and Golledge R.G. 1994: Computational-process modelling of household activity scheduling. Transportation Research $B$ 28: $355-364$.

Gärling T., Kalen T., Romanus T. and Selart M. 1998: Computer simulation of household activity scheduling. Environment and Planning A, 30: 665679.

Gärling T., Gillholm R. and Montgomery W. 1999: The role of anticipated time pressure in activity scheduling. Transportation, 26: 173-191.

Joh C.H., Arentze T.A. and Timmermans H.J.P. 2001: Towards a theory and simulation model of activity-travel rescheduling behavior. Paper pre- sented at the 9th World Conference on Transportation Research, Seoul, July.

Joh C.H., Arentze T.A. and Timmermans H.J.P. 2002: Modelling individuals' activity-travel rescheduling heuristics: Theory and numerical experiments. To appear in Transportation Research Record.

Jones P., Dix M., Clarke M. and Heggie I. 1983: Understanding Travel Behaviour. Gower, Aldershot.

Kitamura R., Yamamoto T. and Fujii S. 1996: A discrete-continuous analysis of time allocation to two types of discretionary activities which ac-

counts for unobserved heterogeneity. In J.B. Lesort (ed.) Transportation and Traffic Theory. Elsevier, Oxford, pp. 431-453.

McNally M.G. 1997: An activity-based micro-simulation model for travel demand forecasting. In: Ettema D. and Timmermans H.J.P. (eds) Activity-based Approaches to Travel Analysis. Pergamon, Oxford, pp. 37-54.

Pred A. 1981: Of paths and projects: Individual behavior and its societal context. In: Cox K.R. and Golledge R.G. (eds) Behavioural Problems in Geography Revisited. Methuen, London, pp. 231-256.

Recker W.W., McNally M.G. and Root G.S. 1985: Travel/activity analysis: Pattern recognition, classification and interpretation. Transportation Research A, 19: 279-296.

Recker W.W. 1995: The household activity pattern problem: General formulation and solution. Transportation Research B, 29: 61-77.

Supernak J. 1988: A dynamic interplay of activities and travel: Analysis of time of day utility profiles. In: Jones P. (ed.) Developments in Dynamic and Activity-based Approaches to Travel Analysis. Avebury, Aldershot, pp. 99-122

Thrift N.J. 1983: On the determination of social action in space and time. Environment and Planning D, 1: 23-57.

Timmermans H.J.P., Arentze T.A. and Joh C.H. 2001: Modelling the effects of anticipated time pressure on the execution of activity programs. Transportation Research Record, 1752: 8-15. 
Appendix I. The results of the simulation of initial schedule optimisation.

Person 1 (high $U^{\max }$, large $\beta$, large $\gamma$ ) $\mathrm{TU}=1455$

\begin{tabular}{lccc}
\hline Activity & Duration & Begin time & End time \\
\hline Sleep & 448 & $23: 22$ & $6: 50$ \\
Pcare & 73 & $6: 50$ & $8: 03$ \\
Work1 & 207 & $8: 03$ & 1130 \\
Lunch & 72 & $11: 30$ & $12: 42$ \\
Dshop & 48 & $12: 42$ & $13: 30$ \\
Work2 & 296 & $13: 30$ & $18: 26$ \\
\hline Dinner & 132 & $18: 26$ & $20: 38$ \\
\hline Leis_O & 164 & $20: 38$ & $23: 22$ \\
\hline \hline
\end{tabular}

\begin{tabular}{lccc} 
Person 2 & (high $U^{\mathrm{max}}$, large $\beta$, small $\gamma$ ) $\mathrm{TU}=1491$ \\
\hline Activity & Duration & Begin time & End time \\
\hline Pcare & 68 & $23: 43$ & $0: 51$ \\
Sleep & 432 & $0: 51$ & $8: 03$ \\
Dshop & 48 & $8: 03$ & $8: 51$ \\
Work1 & 190 & $8: 51$ & $12: 01$ \\
Lunch & 71 & 12.01 & 13.12 \\
Xork2 & 279 & $13: 12$ & 17.51 \\
\hline Dinner & 90 & $17: 51$ & $19: 21$ \\
\hline Leis_O & 138 & $19: 21$ & $21: 39$ \\
\hline Leis_I & 124 & $21: 39$ & $23: 43$ \\
\hline
\end{tabular}

Note: TU denotes total utility.

Note: The activities in grey are assumed out-of-home activities.

Person 3 (high $U^{\max }$, small $\beta$, large $\gamma$ ) TU $=1338$

\begin{tabular}{|c|c|c|c|}
\hline Activity & Duration & Begin time & End time \\
\hline Sleep & 448 & $23: 19$ & $6: 47$ \\
\hline Pcare & 73 & $6: 47$ & $8: 00$ \\
\hline menop & 53 & 8.00 & 853 \\
\hline Work1 1 & 198 & 8.53 & $12 \sqrt{11}$ \\
\hline Luneh & 79 & $=12,11$ & 13.30 \\
\hline Work2 & 296 & 13.30 & 18.26 \\
\hline Leis 0 & 162 & 18.26 & $21: 08$ \\
\hline Dinner & 131 & $21: 08$ & $23: 19$ \\
\hline
\end{tabular}

Person 5 (low $U^{\mathrm{max}}$, large $\beta$, large $\gamma$ ) $\mathrm{TU}=1305$

\begin{tabular}{|c|c|c|c|}
\hline Activity & Duration & Begin time & End time \\
\hline Sleep & 450 & $23: 17$ & $6: 47$ \\
\hline Pcare & 74 & $6: 47$ & $8: 01$ \\
\hline WorkI & 209 & 8.01 & $11: 30$ \\
\hline Eunch & 72 & 11.30 & 12.42 \\
\hline Dshop & 48 & 12,42 & $13: 30$ \\
\hline Work? & 298 & $18 \times 30$ & 1828 \\
\hline Dinner & 135 & $18: 28$ & $20: 43$ \\
\hline Leis_I & 154 & $20: 43$ & $23: 17$ \\
\hline
\end{tabular}

Person 7 (low $U^{\text {max }}$, small $\beta$, large $\gamma$ ) TU $=1188$

\begin{tabular}{|c|c|c|c|}
\hline Activity & Duration & Begin time & End time \\
\hline Sleep & 450 & $23: 16$ & $6: 46$ \\
\hline Pcare & 74 & $6: 46$ & $8: 00$ \\
\hline Dshop & 83 & 800 & 853 \\
\hline Workl & 168 & 853 & 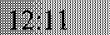 \\
\hline Lunch & 79 & (2) & 13,30 \\
\hline Work2 & 298 & $13 \cdot 30$ & 18.28 \\
\hline Dinner & 134 & $18: 28$ & $20: 42$ \\
\hline Leis_I & 154 & $20: 42$ & $23: 16$ \\
\hline
\end{tabular}

Person 4 (high $U^{\text {max }}$, small $\beta$, small $\gamma$ ) TU $=1383$

\begin{tabular}{|c|c|c|c|}
\hline Activity & Duration & Begin time & End time \\
\hline Pcare & 70 & $0: 09$ & $1: 19$ \\
\hline Sleep & 437 & $1: 19$ & $8: 36$ \\
\hline Work1 & 105 & 8.36 & 1150 \\
\hline Lunen & W6 & 1.5 & 1307 \\
\hline Work2 & 285 & 33.67 & II:52 \\
\hline Dinner & 98 & $17: 52$ & $19: 30$ \\
\hline Gers & 142 & 9939 & $27: 56$ \\
\hline Leis_I & 133 & $21: 56$ & $0: 09$ \\
\hline
\end{tabular}

Person 6 (low $U^{\text {max }}$, large $\beta$, small $\gamma$ ) $\mathrm{TU}=1306$

\begin{tabular}{|c|c|c|c|}
\hline Activity & Duration & Begin time & End time \\
\hline Sleep & 452 & $23: 11$ & $6: 43$ \\
\hline Pcare & 75 & $6: 43$ & $7: 58$ \\
\hline Work1 & 212 & 7.58 & TISO \\
\hline bunch & 72 & I. & 12,42 \\
\hline Dshop: & 48 & $12: 42$ & 13...0 \\
\hline Work2 & 301 & $13: 30$ & $18: 31$ \\
\hline Dinner & 122 & $18: 31$ & $20: 33$ \\
\hline Leis_I & 158 & $20: 33$ & $23: 11$ \\
\hline
\end{tabular}

Person 8 (low $U^{\max }$, small $\beta$, small $\gamma$ ) TU $=1235$

\begin{tabular}{|c|c|c|c|}
\hline Activity & Duration & Begin time & End time \\
\hline Sleep & 439 & $23: 49$ & $7: 08$ \\
\hline Pcare & 70 & $7: 08$ & $8: 18$ \\
\hline Workl & 190 & 8.18 & $11: 35$ \\
\hline Iunch & 78 & 1.35 & 12.58 \\
\hline work2 & 286 & 12.53 & $17: 39$ \\
\hline Leis 0 & 135 & 17.39 & 1954 \\
\hline Dinner & 100 & $19: 54$ & $21: 34$ \\
\hline Leis_I & 135 & $21: 34$ & $23: 49$ \\
\hline
\end{tabular}


Appendix II. The results of the simulation of congestion-induced rescheduling.

\begin{tabular}{|c|c|c|c|c|c|c|c|c|}
\hline \multicolumn{3}{|c|}{$\mathrm{TU}=1434$} & \multicolumn{5}{|c|}{ Person 1 (high $U^{\max }$, large $\beta$, large $\gamma$ ) } & \multirow{2}{*}{$\frac{\mathrm{TU}=1443}{\text { End time }}$} \\
\hline Activity & Duration & Begin time & End time & & Activity & Duration & Begin time & \\
\hline Sleep & 448 & $23: 22$ & $6: 50$ & & Sleep & 448 & $23: 22$ & $6: 50$ \\
\hline Pcare & 73 & $6: 50$ & 8:03 & & Pcare & 73 & $6: 50$ & $8: 03$ \\
\hline Work1 & 177 & $8: 33$ & $11: 30$ & $\Rightarrow$ & Work I & 185 & 8:33 & $11: 38$ \\
\hline Lunch: & 72 & 11,30 & $12: 42$ & & Lunch & 65 & $11: 38$ & $12: 43$ \\
\hline Dshop & 48 & $12: 42$ & $13: 30$ & & Dshop & 47 & $12: 43$ & 13:30 \\
\hline Work2 & 296 & $13: 30$ & 18:26 & & Work2 & 296 & 13:30 & $18: 26$ \\
\hline Dinner & 132 & $18: 26$ & $20: 38$ & & Dinner & 132 & $18: 26$ & $20: 38$ \\
\hline Leis_o & 164 & $20: 38$ & $23: 22$ & & Leis_O & 164 & $20: 38$ & $23: 22$ \\
\hline
\end{tabular}

\begin{tabular}{|c|c|c|c|c|c|c|c|c|}
\hline \multicolumn{3}{|c|}{$T U=1367$} & \multicolumn{5}{|c|}{ Person 2 (high $U^{\max }$, large $\beta$, small $\gamma$ ) } & \multirow{2}{*}{$\frac{T U=1473}{\text { End time }}$} \\
\hline Activity & Duration & Begin time & End time & & Activity & Duration & Begin time & \\
\hline Pcare & 68 & $23: 43$ & $0: 51$ & & Pcare & 68 & $23: 43$ & $0: 51$ \\
\hline Sleep & 432 & $0: 51$ & $8: 03$ & & Sleep & 432 & 0.51 & $8: 03$ \\
\hline Dshop & 18 & $8: 33$ & $8: 51$ & $\Rightarrow$ & Work1 & 189 & $8: 33$ & $11: 42$ \\
\hline Work1 & 190 & $8: 51$ & $12: 01$ & & Lunch & 70 & $11: 42$ & $12: 52$ \\
\hline Lunch & 71 & $12: 04$ & $13: 12$ & & Work? & 278 & $12: 52$ & $17: 30$ \\
\hline Work2 & 279 & $13: 12$ & $17: 51$ & & Dshop: & 47 & $17: 30$ & $18: 17$ \\
\hline Dinner & 90 & $17: 51$ & $19: 21$ & & Dinner & 83 & $18: 17$ & $19: 40$ \\
\hline Leis_o & 138 & $19: 21$ & 21:39 & & Leis_o & 131 & $19: 40$ & $21: 51$ \\
\hline Leis_I & 124 & $21: 39$ & $23: 43$ & & Leis_I & 116 & $21: 51$ & $23: 47$ \\
\hline
\end{tabular}

$\begin{array}{lll}\mathrm{TU}=1334 & \text { Person } 3\left(\text { high } U^{\text {max }} \text {, small } \beta \text {, large } \gamma\right) & \mathrm{TU}=1335\end{array}$

\begin{tabular}{|c|c|c|c|c|c|c|c|c|}
\hline Activity & Duration & Begin time & End time & & Activity & Duration & Begin time & End time \\
\hline Sleep & 448 & $23: 19$ & $6: 47$ & \multirow{7}{*}{$\Rightarrow$} & Sleep & 448 & $23: 19$ & $6: 47$ \\
\hline Pcare & 73 & $6: 47$ & $8: 00$ & & Pcare & 73 & $6: 47$ & 8:00 \\
\hline Dshop & 23 & $8: 30$ & $8: 53$ & & Dshop & $\sqrt{11}$ & $8: 30$ & $8: 41$ \\
\hline Work1 & 198 & $8: 53$ & $12: 11$ & & Work1 & 204 & $8: 41$ & $12: 05$ \\
\hline Lunch & 79 & $12: 11$ & $13: 30$ & & Lunch & 85 & $12: 05$ & $13: 30$ \\
\hline Work2 & 296 & $13: 30$ & $18: 26$ & & Work2 & 296 & $13: 30$ & 18:26 \\
\hline Leis 0 & 162 & $18: 26$ & $21: 08$ & & Leis O & 162 & $18: 26$ & 21:08 \\
\hline Dinner & 131 & $21: 08$ & $23: 19$ & & Dinner & 131 & 21:08 & $23: 19$ \\
\hline
\end{tabular}

\begin{tabular}{|c|c|c|c|c|c|c|c|c|}
\hline \multicolumn{3}{|c|}{$\mathrm{TU}=1284$} & \multicolumn{4}{|c|}{ Person 4 (high $U^{\max }$, small $\beta$, small $\gamma$ ) } & & \multirow{2}{*}{$\begin{array}{l}\mathrm{TU}=1374 \\
\text { End time }\end{array}$} \\
\hline Activity & Duration & Begin time & End time & & Activity & Duration & Begin time & \\
\hline Pcare & 70 & $0: 09$ & $1: 19$ & & Pcare & 70 & $0: 09$ & $1: 19$ \\
\hline Sleep & 437 & $1: 19$ & $8: 36$ & & Sleep & 437 & $1: 19$ & $8: 36$ \\
\hline Work I & 165 & 9.06 & 11:51 & $\Rightarrow$ & Wark & 192 & 906 & $12: 18$ \\
\hline Lanch & 76 & $11: 51$ & $13: 07$ & & Lanch & 72 & $2: 18$ & 13,30 \\
\hline Work2 & 285 & 13.07 & 17.52 & & Work2 & 281 & $3: 30$ & $18: 11$ \\
\hline Dinner & 98 & $17: 52$ & $19: 30$ & & Dinner & 93 & 18:11 & 19:44 \\
\hline Leis O & 146 & $19: 30$ & $21: 56$ & & Leis_O & 141 & $19: 44$ & $22: 05$ \\
\hline Leis_I & 133 & 21:56 & 0:09 & & Leis_I & 127 & $22: 05$ & $0: 12$ \\
\hline
\end{tabular}




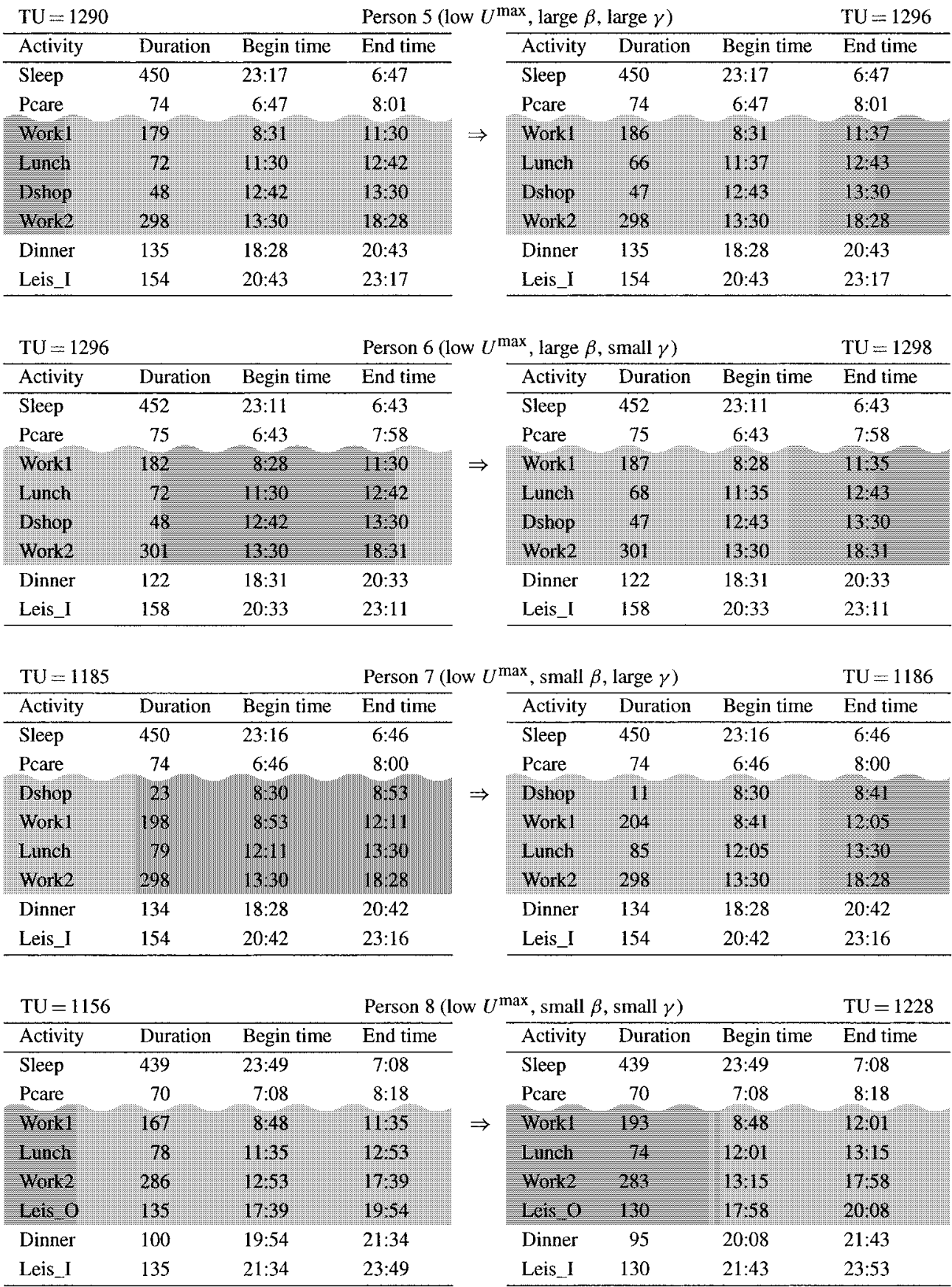

